\begin{tabular}{|c|l|}
\hline Title & Detailed analysis of a single photon source using gated spontaneous parametric downconversion \\
\hline Author(s) & Okamoto, Ryo; Takeuchi, Shigeki; Sasaki, Keiji \\
\hline Citation & $\begin{array}{l}\text { Journal of the Optical Society of A merica B: Optical Phy sics, 22(11), 2393-2401 } \\
\text { https://doi.org/_0.1364/JOSAB.22.002393 }\end{array}$ \\
\hline Issue Date & 2005-11-01 \\
\hline Doc URL & http://hdl.handle.net/2115/49845 \\
\hline Rights & ○ 2005 Optical Society of A merica \\
\hline Type & article \\
\hline File Information & JOSA B22_11_2393-2401.pdf \\
\hline
\end{tabular}

Instructions for use 


\title{
Detailed analysis of a single-photon source using gated spontaneous parametric downconversion
}

\author{
Ryo Okamoto \\ Research Institute for Electronic Science, Hokkaido University, Kita-12 Nishi-6, Kita-ku, Sapporo, \\ Hokkaido, 060-0812 Japan
}

Shigeki Takeuchi

Research Institute for Electronic Science, Hokkaido University, Kita-12 Nishi-6, Kita-ku, Sapporo, Hokkaido, 060-0812 Japan, and Japan Science and Technology Corporation-Precursory Research for Embryonic Science and Technology Project "Light and Control," Kawaguchi Center Building, 4-1-8, Honcho, Kawaguchi-Shi, Saitama 332-0012, Japan

Keiji Sasaki

Research Institute for Electronic Science, Hokkaido University, Kita-12 Nishi-6, Kita-ku, Sapporo, Hokkaido, 060-0812 Japan

Received November 19, 2004; revised manuscript received May 5, 2005; accepted May 18, 2005

\begin{abstract}
The photon-number distribution of an output state from a single-photon source using gated spontaneous parametric downconversion is analyzed in detail. For an improved experimental result of $\{P(0), P(1), P(2)\}$ $=\{0.59,0.40,0.009\}$, it is found that $P(1)$ is restricted by the optical loss of the signal photon path $(62 \%)$ and the cause of nonzero $P(2)$ is the imperfection of the optical shutter for gating. A method by which to reconstruct the photon-number distribution via photon-counting measurement is also presented. () 2005 Optical Society of America

OCIS codes: $270.0270,270.5290,270,6570,030.5260,230.6080$.
\end{abstract}

\section{INTRODUCTION}

The realization of single-photon sources is attracting attention as an indispensable technology for quantum communication and quantum information processing using photons. ${ }^{1,2}$ We herein define an ideal single-photon source as an apparatus that emits only a single photon with $100 \%$ probability in a limited time window at the output port. Therefore, the probability of having one photon in a pulse $P(n=1)$ should be 1 ; otherwise, $P(n \neq 1)=0$. This photon-number distribution (PND) clearly differs from the distribution of weak coherent light with an average photon number equal to $1, P(n)=\exp (-1) / n !$.

Extensive research has been conducted regarding the realization of such single-photon sources. Compared with single-photon sources that use single light emitters, ${ }^{3-7}$ single-photon sources that use parametric downconversion ${ }^{8-15}$ have some advantages, such as roomtemperature operation and wavelength tunability. It should also be noted that the photons are automatically emitted in a certain direction owing to the phasematching condition. ${ }^{16}$ In contrast, controlling the emission of photons from single light emitters, i.e., using a high-finesse cavity, remains difficult.

We recently reported a single-photon source using gated parametric downconversion. ${ }^{17}$ In the process of spontaneous parametric downconversion (SPDC), a pair of photons, an idler photon and signal photon, is created at the same time. In the previous experiment, signal pho- tons were gated by a fast optical shutter according to the detection event of the corresponding idler photons in order to realize a state such that there exists a single photon in a $100 \mu$ s period. Note that the coherence time of the photon pair is much shorter than the repetition time of the operation and the time interval of the photon pair. We reported a PND of $\{P(1), P(2)\}=\{0.27,0.011\}$ at $10 \mathrm{kHz}$ operation. However, to realize an ideal single-photon source with $P(n=1)=1$ and $P(n \neq 1)=0$, we have to clarify the cause of the imperfections in the previous experiment.

In this paper, we present a detailed analysis of the PND of the output state from the single-photon source using gated spontaneous parametric downconversion (GSPDC). Losses in the optical circuit of the source and the imperfections of the trigger-photon detector and the optical shutter are considered in the present analysis. The analysis results corresponded well to our updated result of $\{\mathrm{P}(1), \mathrm{P}(2)\}=\{0.40,0.009\}$ at $10 \mathrm{kHz}$ and $\{\mathrm{P}(1), \mathrm{P}(2)\}$ $=\{0.39,0.019\}$ at $50 \mathrm{kHz}$. In addition, we investigated a method by which to reconstruct the PND from the measurement result given by the nonideal detection system, which was not fully discussed in the previous paper. ${ }^{17}$ Nonunity effective quantum efficiency, afterpulses, dark counts, and the dead time of the detection system are considered quantitatively the imperfect factors in the reconstruction of the PND.

This paper is organized as follows. Section 2 presents a description of the experimental setup. Section 3 describes 


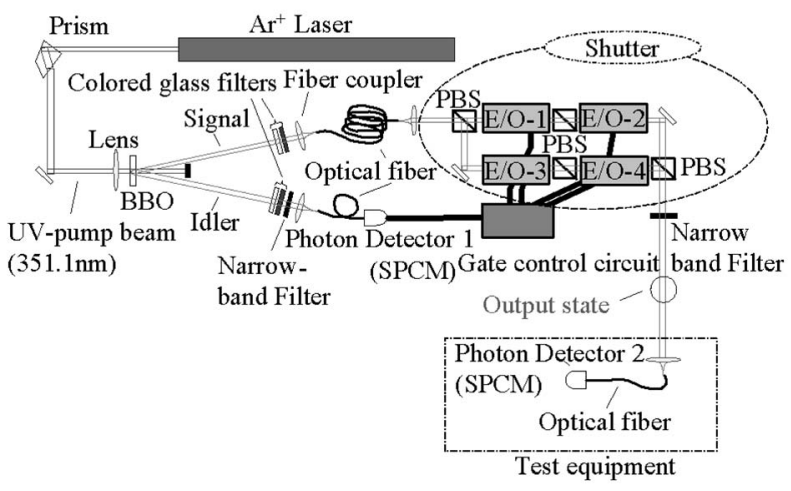

Fig. 1. Schematic diagram of the experimental setup. When a pump beam is incident to a BBO crystal, SPDC occurs. Through the process of SPDC, signal photons and idler photons are generated at the same time. The idler photons are detected, and the signal photons are guided into an optical fiber. The gating circuit then operates the shutter in order to output only one signal photon for a fixed period.

the measurement scheme and the photon-count distribution results, and Section 4 describes the method used to obtain a PND from the photon-count distribution by using the compensation of the detection system. A detailed analysis of the obtained PND in terms of the imperfection is given in Section 5. Finally, conclusions are presented in Section 6.

\section{EXPERIMENTAL SETUP}

Figure 1 shows a schematic diagram of the experimental setup of a single-photon source using G-SPDC. This system contains three key components: a setup for SPDC and idler photon detection, a shutter, and a gate control circuit. In the following, we will explain each of these components.

\section{A. Setup for Spontaneous Parametric Downconversion and Idler Photon Detection}

A $5 \mathrm{~mm}$ thick type-II beta-barium borate (BBO) crystal was pumped by an argon-ion laser at a wavelength of $351.1 \mathrm{~nm}$. We adopted a degenerate phase-matching condition for the twin-beam generation at a central wavelength of $702.2 \mathrm{~nm} .{ }^{16}$ To optimize the photon-pair collection efficiency, we placed a convex lens for the pump beam before the BBO crystal so that the pump beam was focused in the middle of two fiber couplers. ${ }^{18}$ After we removed the scattered pump light by using two colored glass filters and a narrowband filter, each of the idler photons and the signal photons was guided to multimode fiber couplers. These colored glass filters cut off the light whose wavelength is shorter than $640 \mathrm{~nm}$. The narrowband filter has a center wavelength of $702.2 \mathrm{~nm}$ and a bandwidth of $4 \mathrm{~nm}$. The idler photon was then detected by photon detector 1 , which is an avalanche photodiode operated in Geiger mode (SPCM-AQ-FC, Perkin Elmer). During the time it takes to open the shutter after the detection of the idler photon, which was $150 \mathrm{~ns}$ in our experiment, the signal photon should be maintained with a small loss. For this purpose, we adopted an optical delay line using a multimode fiber (length: $35 \mathrm{~m}$ ). Note that for the signal photons we used two colored glass filters before the multimode fiber coupler.

\section{B. Shutter}

The shutter consists of four electro-optic (E/O) modulators and four polarizing beam splitters (PBSs) (Fig. 1). In our experiment, the polarization at the output of the optical delay line (multimode fiber) is random owing to the use of multimode fiber. In this situation, a classic Kerr shutter configuration, such that an E/O modulator is placed between two polarizers, may lose at least $50 \%$ of input photons. For obtaining high $P(1)$, however, the transmission of the shutter should be as high as possible. Therefore, we adopted a configuration in which the signal photon was sent along one of two paths, according to its polarization, by a PBS, and its polarization was rotated by the E/O modulators in each path. The output in each path was then merged by the second PBS. To minimize the shutter open time and avoid the restriction due to the repetition rate $(1 \mathrm{MHz})$ of the $\mathrm{E} / \mathrm{O}$ modulator drivers (LIV-8, Linos), we used two E/O modulators sequentially to control the polarization of the photons. With this configuration, we achieved $83 \%$ transmittance for an open shutter. In Subsection 2.C, we explain how this optical shutter is controlled according to the detection pulse of the idler photon.

\section{Gate Control Circuit}

The timing chart of the electric signals used to control the optical shutter circuit is shown in Fig. 2. The cycle of the clock that was used to determine the optical pulse duration $\tau_{p}$ was given by a function generator (DS345, Stanford Research Systems). Intermissions $(2 \mu \mathrm{s})$ are needed owing to the repetition rate of the $\mathrm{E} / \mathrm{O}$ modulator driver (1 MHz). Each E/O modulator followed by a PBS can be

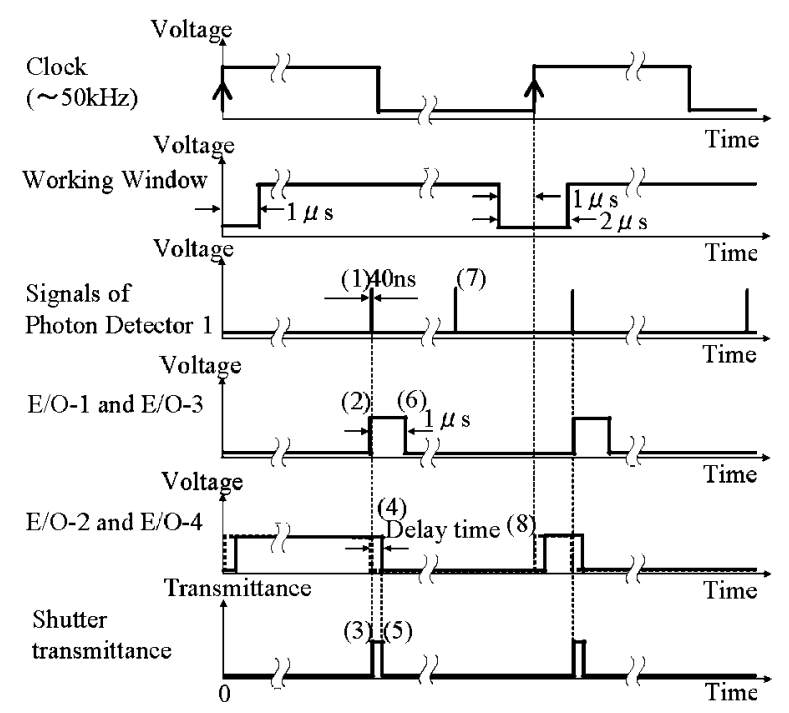

Fig. 2. Timing chart of the shutter controlled by the gate control circuit. When the working window is set high, the gate control circuit reacts. Following the first photon detection pulse of photon detector 1 during one clock cycle, the gate control circuit opens the shutter for a short time. To minimize the shutter open time, the gate control circuit controls the first set of $\mathrm{E} / \mathrm{O}$ modulators (E/O-1 and E/O-3) and the second set of E/O modulators (E/O-2 and E/O-4) separately. 
considered a conventional Kerr shutter. When the voltage to an E/O modulator is at the high (low) level, the Kerr shutter is open (closed). The gate control circuit controls the first two E/O modulators (E/O-1 and E/O-3) and the second two E/O modulators (E/O-2 and E/O-4) independently.

At the time origin, the voltages to the first set of $\mathrm{E} / \mathrm{O}$ modulators and the voltages of the second set of E/Os are at the low level (closed). After a short delay time, the control circuit opens the second set of E/O modulators. Reacting to the first detection pulse in a clock cycle (1), the gate control circuit increases the voltages of the first set of E/Os to the high level (open) (2). The state of the entire shutter is then open (3). After a delay time (33 ns), the control circuit turns off the second set of $\mathrm{E} / \mathrm{O}$ modulators (4), which means that the entire shutter is now closed (5). To minimize the leaking of photons through the closed shutter, we also turned off the first set of E/O modulators after an appropriate period of time $(1 \mu \mathrm{s})(6)$. For the detection pulse after the first detection pulse in the clock pulse (7), the gate control circuit does not react, so that the signal photons corresponding to those detection pulses are blocked. The above procedure is then repeated for the remaining clock cycles.

\section{MEASUREMENT OF PHOTON-NUMBER DISTRIBUTION}

Before the experiment to obtain the PND of the output state, we measured the single-count and coincidencecount rates using photon detector 1 and photon detector 2 when the shutters were opened. When the power of the pumping laser was $53 \mathrm{~mW}$, the single-count rates of the idler photon and the signal photon and the coincidencecount rate were $1.19 \times 10^{5}, 8.76 \times 10^{4}$, and $2.81 \times 10^{4}$ counts per second (cps), respectively.

To obtain the PND, we counted the number of photons in each optical pulse by using photon detector 2 . The single-photon counting module (SPCM) generates an electric pulse (pulse width $40 \mathrm{~ns}$ ) when a photon is detected. For the purpose of removing the afterpulses, ${ }^{19}$ we expanded the pulse width of the SPCM from $40 \mathrm{~ns}$ to $1.5 \mu \mathrm{s}$ via a digital delay and a pulse generator (DG535, Stanford Research Systems). Before this treatment, the probability of having afterpulses within $100 \mu \mathrm{s}$ (optical pulse duration) was $2 \times 10^{-3}$. After this treatment, the probability decreased to $3 \times 10^{-6}$. As a result, the number of afterpulses was $0.01 \mathrm{cps}$, which is smaller than the standard deviation of the intrinsic dark count of the detector (10 cps).

The measurement result of the photon-count distribution is as follows, where $N(n)$ is the number of photoncount pulses having $n$ counts in an optical pulse: $N(0)$, 76,$194 ; N(1), 23,305 ; N(2), 493 ; N(3), 8$; and $N(n>4), 0$. The pulse duration was $100 \mu \mathrm{s}$. The total number of optical pulses measured was $1.0 \times 10^{5}$.

The photon-count probability $P(n)$ can be obtained by dividing $N(n)$ by the total number of optical pulses. The distribution of the photon-count probability is shown in Table 1. The number of significant digits of $P(n)$ is determined by the fluctuation of each $N(n)$, which is defined by $[N(n)]^{1 / 2}$. In this experiment, $N(3)$ and $N(4)$ are of the
Table 1. Photon-Count Probability Distribution

\begin{tabular}{ccc}
\hline$P(0)^{a}$ & $P(1)$ & $P(2)$ \\
\hline 0.76 & 0.23 & 0.005 \\
\hline
\end{tabular}

${ }^{a} P(n)$ is the photon-count probability of having $n$ counts in an optical pulse.

same order as their fluctuation. Therefore, in the following analysis, we will focus our attention on $P(n)$ with $n$ $\leqslant 2$. In Section 4, we discuss how to compensate for the imperfections, such as the nonunity quantum efficiency, of the detection system for estimating the PND of the output state.

\section{ESTIMATION OF THE PHOTON-NUMBER DISTRIBUTION}

In this section, we try to estimate the real PND in the output state. The photon-count distribution obtained in Section 3 is affected by the nonideal characteristics of the detection system: dead time, dark count, and losses. The sequence of physical processes corresponding to these characteristics may be regarded as follows.

(1) Loss: A signal photon may not be detected owing to loss before the detector or nonunity quantum efficiency.

(2) Dark count: The detector may cause some additional noise signals.

(3) Dead time: Some of the electric signal pulses are missed by the electric pulse counter because of nonzero pulse width.

These effects must be compensated for in reverse order of their occurrence in order to obtain the PND. Therefore, the compensation should be performed in the order of dead time, dark count, and losses.

\section{A. Effect of Dead Time}

To decrease the effect of afterpulses, we extended the electric signal pulse duration $\tau_{d}$ from $40 \mathrm{~ns}$ to $1.5 \mu \mathrm{s}$, as is explained in Section 3. However, if a following signal pulse is within the extended time duration, it cannot be detected or lost. In this sense, $\tau_{d}$ can be considered the dead time of the detection apparatus. The effect of this dead time on the distribution of signal pulses can be written as follows:

$$
\begin{gathered}
P(0)=P^{\prime}(0)+P_{l d 11} P^{\prime}(1), \\
P(1)=\left(1-P_{l d 11}\right) P^{\prime}(1)+P_{l d 21} P^{\prime}(2), \\
P(2)=\left(1-P_{l d 21}\right) P^{\prime}(2),
\end{gathered}
$$

where $P^{\prime}(n)$ and $P(n)$ are the probabilities of having $n$ signal pulses in an optical pulse without and with the effect of dead time, respectively. $P_{l d 11}$ and $P_{l d 21}$ are the respective probabilities for (1) the existence of only one signal pulse in the optical pulse, which is then lost owing to the dead time, and (2) the existence of two signal pulses, one of which is then lost. In the following, we derive $P_{l d 11}$ and $P_{l d 21}$ on the basis of the assumption that the signal pulses are uniformly distributed in the optical pulse time dura- 
tion $\tau_{p}$. In our experiments, the ratio $\gamma \equiv \tau_{d} / \tau_{p}<0.08$ and $P(1) \gg P(2)$. Therefore, we derive the approximated value $P_{l d 11}$ and $P_{l d 21}$ to the second order and the first order, respectively, of $\gamma$.

First, we investigate the factor $P_{l d 11}$. This probability corresponds to the case whereby only one signal pulse $A$ exists in the optical pulse but is lost owing to the dead time. This situation may occur only when the signal pulse $A$ is in the dead time of another signal pulse $B$, which is in the previous optical pulse. This can occur only when the following condition is satisfied:

$$
\tau_{p}-\tau_{d} \leqslant t_{p}<\tau_{p}
$$

where $t_{p}$ is the position (time) of the signal pulse $B$ in the previous optical pulse. In our experiment, there is no time interval between optical pulses. Therefore, the dead time in the present optical pulse due to the signal pulse $B$ is given by $t_{p}-\left(\tau_{p}-\tau_{d}\right)$. Here, let us assume that we have only one signal pulse in the previous optical pulse. The probability of having the signal pulse in a small time window $\mathrm{d} t$ is given by $\mathrm{d} t / \tau_{p}$. Therefore, the probability of losing the signal pulse in the present optical pulse is as follows:

$$
\int_{\tau_{p}-\tau_{d}}^{\tau_{p}} \frac{1}{\tau_{p}} \int_{0}^{t_{p}-\left(\tau_{p}-\tau_{d}\right)} \frac{1}{\tau_{p}} \mathrm{~d} t_{c} \mathrm{~d} t_{p}=\frac{\gamma^{2}}{2},
$$

where $t_{c}$ is the position (time) of the signal pulse $A$.

Let us now consider the case in which we have $n$ signal pulses in the previous optical pulse. In this case, the probability of having one of the signal pulses in a small time window $\mathrm{d} t$ in the previous optical pulse is given by $n \mathrm{~d} t / \tau_{p}$ by neglecting the higher-order term. Taking into account the fact that the probability of counting $n$ signal pulses is now given by $P(n)$, we can write $P_{d l 11}$ as follows:

$$
\begin{aligned}
P_{d l 11} & =\sum_{n=0}^{\infty} P(n) \int_{\tau_{p}-\tau_{d}}^{\tau_{p}} \frac{n}{\tau_{p}} \int_{0}^{t_{p}-\left(\tau_{p}-\tau_{d}\right)} \frac{1}{\tau_{p}} \mathrm{~d} t_{c} \mathrm{~d} t_{p} \\
& =\int_{\tau_{p}-\tau_{d}}^{\tau_{p}} \frac{\bar{n}}{\tau_{p}} \int_{0}^{t_{p}-\left(\tau_{p}-\tau_{d}\right)} \frac{1}{\tau_{p}} \mathrm{~d} t_{c} \mathrm{~d} t_{p}=\frac{\bar{n} \gamma^{2}}{2},
\end{aligned}
$$

where $\bar{n} \equiv \sum_{n=0}^{\infty} n P(n)$ is the measured average number of signal pulses in an optical pulse.

Second, we study the factor $P_{l d 21}$. Although two signal pulses (say, pulse $A$ and pulse $B$ ) originally existed in the optical pulse, one of the two pulses is lost owing to the dead time. In Section 5, we will consider only the case in which one of the pulses is in the dead time of the other pulse and is lost. Note that we neglect the case in which the signal pulse is lost owing to the dead time of the signal pulses in the previous optical pulse, because the probability of this case is of the order of $\gamma^{2}$ or higher.

The probability that pulse $B$ is in the dead time of pulse $A$ and lost is given as follows:

$$
\begin{array}{r}
\int_{0}^{\tau_{p}-\tau_{d}} \frac{1}{\tau_{p}} \int_{t_{A}}^{t_{A}+\tau_{d}} \frac{1}{\tau_{p}} \mathrm{~d} t_{B} \mathrm{~d} t_{A}+\int_{\tau_{p}-\tau_{d}}^{\tau_{p}} \frac{1}{\tau_{p}} \int_{t_{A}}^{\tau_{p}} \frac{1}{\tau_{p}} \mathrm{~d} t_{B} \mathrm{~d} t_{A} \\
=\gamma-\frac{\gamma^{2}}{2} \approx \gamma
\end{array}
$$

where $t_{A}$ and $t_{B}$ are the position (time) of the pulses $A$ and $B$. The probability that pulse $A$ is in the dead time of pulse $B$ is given by the same equation. Therefore, the factor $P_{l d 21}$ is

$$
P_{l d 21}=2 \gamma
$$

From Eqs. (1), (4), and (6), one can find the equations between $\{P(n)\}$ and $\left\{P^{\prime}(n)\right\}$ as follows:

$$
\begin{gathered}
P(0)=P^{\prime}(0)+\frac{\bar{n} \gamma^{2}}{2} P^{\prime}(1), \\
P(1)=\left(1-\frac{\bar{n} \gamma^{2}}{2}\right) P^{\prime}(1)+2 \gamma P^{\prime}(2), \\
P(2)=(1-2 \gamma) P^{\prime}(2),
\end{gathered}
$$

Here, we have analyzed the effect of dead time under the assumption that the signal pulses are uniformly distributed in the optical pulse time duration. However, as discussed in the next section, we found that the signal pulses tend to bunch up because background parametric fluorescence, which cannot be distinguished from the signal photons that are the partners of the first-detected idler photons in the optical pulse duration $\tau_{p}$, leaks through the open shutter. We present a method by which to compensate for the effect of dead time while taking this bunching phenomena into account in Appendix A.

\section{B. Effect of Dark Count}

Here, we assume that the probability $P^{\prime \prime}(m)$ of having $m$ counts per optical pulse due to photons sent to the detector and the probability of having $l$ dark counts per optical pulse are independent. In this case, the joint probability of having $n$ signal counts and $m$ dark counts can be simply given by

$$
P^{\prime \prime}(m) P_{d}(l)
$$

When we have $n$ counts per optical pulse, the sum of the signal counts and the dark counts should be $n$. Therefore, the probability $P^{\prime}(n)$ of having $n$ counts is given as follows:

$$
P^{\prime}(n)=\sum_{m \leqslant n} P_{d}(n-m) P^{\prime \prime}(m)
$$

Because dark counts are caused by stochastic processes, the dark-count distribution $\left\{P_{d}(n)\right\}$ can be approximated by a Poisson distribution $P_{d}(n)=\alpha^{n} \exp (-\alpha) / n$ !, where $\alpha$ is the average number of dark counts per optical pulse. The above equation then reads as ${ }^{8}$

$$
P^{\prime}(n)=\sum_{m \leqslant n} \frac{\alpha^{n-m} \exp (-\alpha)}{(n-m) !} P^{\prime \prime}(m) .
$$

\section{Loss of Photons in the Detection System}

Some photons are lost in the optical components of the detection system with the probability $(1-T)$, where $T$ is the effective transmittance of the optics used in the system. Some photons are not counted by the detector because of the nonunity quantum efficiency $\eta_{D}$ of the photon detec- 
tor. The probability that a signal photon is finally counted by the detection system is called the effective quantum efficiency $\eta=\eta_{D} T$. The relationship between the probability $P^{\prime \prime \prime}(n)$ of having $n$ counts during an optical pulse without loss and the probability $P^{\prime \prime}(n)$ of having $n$ counts during an optical pulse with a reasonable loss is as follows ${ }^{8}$ :

$$
P^{\prime \prime}(n)=\sum_{m \geqslant n}{ }_{m} C_{n} \eta^{n}(1-\eta)^{m-n} P^{\prime \prime \prime}(m),
$$

where ${ }_{m} C_{n}$ is the combinations of $m$ things taken $n$ at a time.

\section{Photon-Number Distribution of Output}

We estimate the PND [Table 2, row (a)] at the output from the photon-count probability distribution (Table 1) by using expressions (1)-(11). The calculated PND of weak coherent light with average photon number [Table 2, row (b)] and the same $\mathrm{P}(2)$ [Table 2, row (c)] are also shown. The parameters of the detection system are shown in Table 3. In Table 4, the details of the effective quantum efficiency are given. The values of the multimode fiber coupler and photon detector 2 are measured independently by using a laser diode with a wavelength of 693 nm. The probability $P(1)$ is $0.40 \pm 0.03$, which is more than 1.5 times larger than the previously reported value (0.27). ${ }^{17}$ The probability $P(2)$ is $0.007 \pm 0.003$. The average photon number of the PND of the output is 0.41 . Here,

Table 2. Photon-Number Distribution ${ }^{a}$

\begin{tabular}{llll}
\hline & \multicolumn{1}{c}{$P(0)$} & \multicolumn{1}{c}{$P(1)$} & \multicolumn{1}{c}{$P(2)$} \\
\hline (a) & $0.59( \pm 0.03)$ & $0.40( \pm 0.03)$ & $0.007( \pm 0.003)$ \\
(b) & 0.67 & 0.27 & 0.056 \\
(c) & 0.88 & 0.11 & 0.007 \\
\hline
\end{tabular}

${ }^{a}$ Row (a) shows the PND that was estimated from the original data. Row (b) shows the Poisson distribution with the same average photon number as in row (a). Row (c) shows the Poisson distribution with the same $P(2)$ as in row (a).

Table 3. Parameters of the Detection System

\begin{tabular}{lc}
\hline Parameter & Value \\
\hline Dark count (cps) & $120 \pm 10$ \\
Effective quantum efficiency $(\%)$ & $56.3 \pm 5$ \\
$\left\langle P_{\mathrm{BG}}(n)\right\rangle^{a}\left(\right.$ count/shutter open time) $\times 10^{-3}$ & $2.87 \pm 0.02$ \\
\hline
\end{tabular}

${ }^{a}\left\langle P_{\mathrm{BG}}\right\rangle(n)$ is the average number of detection pulses of background parametric fluorescence (see Appendix A).
$P(1)$ of the G-SPDC single-photon source is three times as large as that of the weak coherent light with the same $P(2)$. Note that the PND in Table 2, row (a) is calculated under the assumption that the signal pulses are uniformly distributed in the optical pulse duration time.

The PND $\{P(0), P(1), P(2)\}$ is also estimated using Eqs. (A5), (A10), and (A11) in Appendix A, in which the bunching phenomena of single pulses is considered. The result is shown in Table 5. $P(2)$ is $20 \%$ larger than that listed in Table 2, row (a). The signal pulse tends to be in dead time owing to the bunching effect. Thus, it follows that $P(2)$ compensated for the bunching effect is larger than that without compensation. In the following discussion, we use the PND obtained from this compensation.

In principle, $\{P(0), P(1), P(2)\}$ should be $\{0,1,0\}$ with an ideal G-SPDC single-photon source. However the experimental data listed in Tables 2, row (a) and 5 are different from the ideal distribution. In Section 5 we will analyze the imperfection of the experimental results.

\section{ANALYSIS OF THE IMPERFECTION OF THE EXPERIMENTAL RESULT FOR A PHOTON-NUMBER DISTRIBUTION}

In principle, we can construct an ideal single-photon source using the G-SPDC method with a PND of $\{P(n$ $\neq 1)=0, P(1)=1\}$. However, the PND obtained in Section 4 was $\{P(0)=0.59 \pm 0.03, P(1)=0.40 \pm 0.03$, and $P(2)$ $=0.009 \pm 0.003\}$, which differs from the ideal case. In this section, we attempt to theoretically predict the PNDs while considering the possible causes of imperfection, such as losses in the optical circuit of the source, imperfection of the trigger-photon detector, and the imperfection of the optical shutter. We then compare the estimated PNDs with the experimental results.

The measured PND, which is a set of probabilities, can be considered the sum of two different PNDs. One is the PND of the signal photons that are the partners of the first-detected idler photons in the optical pulse duration $\tau_{p}$. We name this PND the PND of the selected signal photons. The other is the PND due to background photons. We first characterize these two PNDs independently and then estimate the total PND of the output photon.

\section{A. Photon-Number Distribution Due to Selected Signal Photons}

The PND of selected signal photons does not include the possibility of having more than one photon because these

Table 4. Effective Quantum Efficiency of the Detection System

\begin{tabular}{lc}
\hline Component & Transmittance, Quantum Efficiency (\%) $^{a}$ \\
\hline Mirror (catalog value) & 99.0 \\
Two lenses (catalog value) (one with broadband antireflection coating, one without & 91.1 \\
$\quad$ antireflection coating) & $83.2 \pm 0.2$ \\
Multimode fiber coupler (measured value) & $75.0 \pm 5$ \\
Photon detector 2 (SPCM) (measured value) & $56.3 \pm 5$ \\
Effective quantum efficiency & \\
\hline
\end{tabular}

\footnotetext{
${ }^{a}$ Measured values are obtained using a laser diode with a wavelength of $693 \mathrm{~nm}$.
} 
photons are the partners of the first-detected idler photons in the optical pulse duration.

To have selected signal photon emission, one must detect at least one idler photon during the time duration $\tau_{p}$. The probability $P_{i}(n)$ of detecting $n$ idler photons in a pulse duration $\tau_{p}$ can be approximated by a Poisson distribution because photon pairs are emitted into multimodes by the SPDC process in our experiment. ${ }^{20}$ Thus, the $P_{i}(0)$ is written as

$$
P_{i}(0)=\exp \left(-N_{1}\right)
$$

where $N_{1}$ is the average single counts of the idler photon in an optical pulse duration. The probability of having at least one idler photon in an optical pulse duration is given by $1-P_{i}(0)$, which can be as close as unity when $N_{1}$ is sufficiently large. Such a condition can be achieved by one's adjusting the pump power.

Even when more than one idler photon is detected during $\tau_{p}$, the selected signal photon may be lost in the optical circuit in the signal photon generator. When the overall transmittance for selected signal photons is given by $T s$, the probability $P_{g}(1)$ of having one photon at the output port is given as follows:

$$
P_{g}(1)=\left[1-P_{i}(0)\right] T s .
$$

To estimate the overall transmittance Ts experimentally, we can use the absolute quantum efficiency method. ${ }^{21}$ According to this method, Ts is given as follows:

$$
T s=\frac{\eta_{2}}{\eta_{m}},
$$

where the $\eta_{m}$ and $\eta_{2}$ are the photon collection efficiency of the detection system and the absolute quantum efficiency of photon detector 2 . In our experiment, we used the value for $\eta_{m}$ shown in Table 4 .

After all, $\left\{P_{g}(n)\right\}$ is given as follows:

$$
\begin{gathered}
P_{g}(0)=1-\left[1-P_{i}(0)\right] T s, \\
P_{g}(1)=\left[1-P_{i}(0)\right] T s, \\
P_{g}(2)=0 .
\end{gathered}
$$

\section{B. Leakage of Photons}

Leakage of unnecessary parametric fluorescence may occur because the shutter used in our experiment is not perfect with respect to the noninfinitesimal shutter open time (33 ns) or the leakage of photons, albeit small, through the closed shutter.

The average photon number of the leaking parametric fluorescence is given as follows:

$$
\alpha_{l}=\frac{N_{2}^{\prime}-N_{c . g}}{\eta_{m}},
$$

where $N_{2}^{\prime}$ is the average single counts of the signal photon in $\tau_{p}$ when the shutter is appropriately operated and $N_{c . g}$ is the average coincidence counts between the signal photon and the first-detected idler photon in $\tau_{p}$. The numerator is the single counts of the leaking parametric fluores- cence when the shutter is appropriately operated. Here, $N_{2}^{\prime}-N_{c . g}$ contains the loss of the detection system, so that these values are divided by the collection efficiency of the detection system $\eta_{m}$. The PND of the leaking parametric fluorescence can be approximated by a Poisson distribution as follows:

$$
P_{l}(n)=\frac{\alpha_{l}^{n} \exp \left(-\alpha_{l}\right)}{n !} .
$$

\section{Photon-Number Distribution of Output Photon}

The probability $P(n)$ of having $n$ photons during an optical pulse duration can be written using $P_{g}(n)$ and $P_{l}(n)$ as follows:

$$
P(n)=\sum_{l=0, m=0}^{l+m=n} P_{g}(l) P_{l}(m),
$$

for $n=0,1,2$.

\section{Comparison of Estimated and Measured Photon- Number Distributions}

Using Eqs. (15)-(17), (19), and (20), we can reconstruct the PND of the output by the parameters $T s, P_{i}(0)$, and $\alpha_{l}$. First, we attempt to reconstruct the result of Table 2, row (a). The measured values for $T s, P_{i}(0)$, and $\alpha_{l}$ were $0.38,6.6 \times 10^{-6}$, and 0.016 , respectively. Thus, the estimated PND is $\{P(1)=0.38 \pm 0.01, P(2)=0.006 \pm 0.005\}$, which is in accordance, within the error range, with the experimental data of Table 5. In this way, $P(1)$ is limited by $T s$, and $P(2)$ is due to $\alpha_{l}$ in this experiment.

To check the validity of the analysis given here, we measured the PNDs of the output state from the G-SPDC source operated with three different sets of pump powers and repetition rates: row (a) $53 \mathrm{~mW}, 10 \mathrm{kHz}$; row (b) 53 $\mathrm{mW}, 50 \mathrm{kHz}$; and row (c) $170 \mathrm{~mW}, 50 \mathrm{kHz}$. Table 6 shows the measurement results of the PND of the outputs and the estimated results of the corresponding PND using the above equations. Table 7 shows the parameters used to obtain the estimated results.

When the repetition rate was increased from 10 to 50 $\mathrm{kHz}$ while the pump laser intensity was kept constant (53 $\mathrm{mW}), P(1)$ decreased from $0.40 \pm 0.03$ [Table 6, row (a)] to $0.32 \pm 0.02$ [Table 6 , row (b)]. This degradation of $P(1)$ is mainly due to the decrease in the average number of idler photon detection events per optical pulse, $\left\langle n_{\text {idler }}\right\rangle$. In that $\left\langle n_{\text {idler }}\right\rangle$ at $50 \mathrm{kHz}$ was 2.4 , which is one fifth of $\left\langle n_{\text {idler }}\right\rangle$ at 10 $\mathrm{kHz}$, the probability of having no idler photon detection in an optical pulse becomes significant. The PNDs $\{P(1), P(2)\}$ are well reproduced by the theoretical predictions.

To increase $P(1)$ at $50 \mathrm{kHz}$ to the level recorded at 10 $\mathrm{kHz}$, we increased the pump laser intensity from 53 to $170 \mathrm{~mW}$ [Table 6, row (c)]. The measurement result of

Table 5. Photon-Number Distribution Considering the Analysis of Appendix A

\begin{tabular}{ccc}
\hline$P(0)$ & $P(1)$ & $P(2)$ \\
\hline $0.59( \pm 0.03)$ & $0.40( \pm 0.03)$ & $0.009( \pm 0.003)$ \\
\hline
\end{tabular}


Table 6. Measured and Estimated Values of Photon-Number Distribution

\begin{tabular}{lccrrr}
\hline & & \multicolumn{2}{c}{ Measured Value } & \multicolumn{2}{c}{ Estimated Value } \\
& Operation Condition $^{a}$ & $\mathrm{P}(1)$ & $\mathrm{P}(2)$ & $\mathrm{P}(1)$ & $0.38( \pm 0.01)$ \\
(a) & $53 \mathrm{~mW}, 10 \mathrm{kHz}$ & $0.40( \pm 0.03)$ & $0.009( \pm 0.003)$ & $0.006( \pm 0.005)$ \\
(b) & $53 \mathrm{~mW}, 50 \mathrm{kHz}$ & $0.32( \pm 0.02)$ & $0.011( \pm 0.002)$ & $0.01)$ & $0.007( \pm 0.002)$ \\
(c) & $170 \mathrm{~mW}, 50 \mathrm{kHz}$ & $0.39( \pm 0.03)$ & $0.019( \pm 0.002)$ & $0.39( \pm 0.01)$ & $0.017( \pm 0.001)$ \\
\hline
\end{tabular}

${ }^{a}$ Pump powers are 53 and $170 \mathrm{~mW}$, and 10 and $50 \mathrm{kH}$ are repetition rates.

Table 7. Parameters for Estimation

\begin{tabular}{lcccc}
\hline & Operation Condition $^{a}$ & Ts & $P_{i}(0)$ & $\alpha_{l}$ \\
\hline (a) & $53 \mathrm{~mW}, 10 \mathrm{kHz}$ & 0.38 & $6.6 \times 10^{-6}$ & 0.016 \\
(b) & $53 \mathrm{~mW}, 50 \mathrm{kHz}$ & 0.37 & 0.10 & 0.021 \\
(c) & $170 \mathrm{~mW}, 50 \mathrm{kHz}$ & 0.38 & $3 \times 10^{-3}$ & 0.045 \\
\hline
\end{tabular}

${ }^{a}$ Pump powers are 53 and $170 \mathrm{~mW}$, and 10 and $50 \mathrm{kH}$ are repetition rates.

$P(1)$ is $0.39 \pm 0.03$, which is as high as the previous value [Table 6, row (a)]. In this experiment, $\left\langle n_{\text {idler }}\right\rangle$ is increased from 2.4 [Table 6 , row (b)] to 7.7, and the probability of having no idler photon detection was again well suppressed. Note that the theoretical calculations for PND [Table 6, row (c)] correspond to the experimental results within the errors.

\section{CONCLUSION}

Our goal is to realize a photon source that generates a single photon in a given time duration. In this paper, we discussed in detail the reconstruction of the photonnumber distribution (PND) at the output state using a conventional photon-counting device. The estimated PNDs of the collimated output states are $\{P(1), P(2)\}$ $=\{0.40,0.009\}$ and $\{0.39,0.019\}$ for 10 and $50 \mathrm{kHz}$ repetition rates, respectively, where $P(n)$ denotes the probability of finding $n$ photons in a pulse duration. These $P(1) \mathrm{s}$ are 1.5 times larger than those in our previous report. ${ }^{17}$ We also presented a detailed analysis of the obtained PNDs. The PNDs are well explained in terms of $T s, P_{i}(0)$, and $\alpha_{l}$, where $T s$ is the overall transmittance for the signal photons, $P_{i}(0)$ is the probability of not detecting idler photons in pulse duration $\tau_{p}$, and $\alpha_{l}$ is the average photon number of the leaking parametric fluorescence. We also confirmed that the increase of $P(1)$ is mainly due to the higher transmittance of the frequency filter used in the modified setup. We also found that the $P(1)$ s of our modified source are still restricted by the optical losses due to the coupling efficiency of parametric fluorescence, the transmittance of the shutter, and the narrowband filter. The main cause of $P(2)$ s was found to be nonzero transmittance of the optical shutter when the shutter was closed.

In the reconstruction of the PNDs, we first assumed a uniform distribution of photon-emission time in a pulse duration and took into account the effects of dead time, afterpulses, dark counts, and the limited effective quantum efficiency of the detection apparatus. We also performed a further analysis in which the nonuniform distribution of the photon-emission time due to the mechanism of the gating optical circuit is considered and found that the reconstructed PNDs are approximately equivalent to those using the former simple assumption.

The complete analysis of PNDs presented herein can be used to design a photon source for a specific application requiring high-probability single-photon emission.

Room-temperature operation, long lifetime, and wavelength tunability of the source may be favorable for such applications.

\section{APPENDIX A: EFFECT OF DEAD TIME ON THE ESTIMATED PHOTON-NUMBER DISTRIBUTION CONSIDERING THE PHOTON ARRIVAL TIME}

In Section 4, we assumed a uniform distribution of signal photon arrival time in optical pulse duration when we discussed the effect of dead time. This assumption may be too simple when we consider the gating mechanism in our single-photon source: The photons leaking through the shutter tend to bunch up during the shutter open time. Therefore, in this appendix, we discuss the effect of dead time on the PND, taking the above effect into account.

First, let us show that the effect of dead time on the background photons through the closed shutter is negligible in our experiment. As shown in Table 7, $T s\left[1-P_{i}(0)\right]$, which corresponds to the number of signal photons corresponding to the detected idler photons (gated signal photons), is much larger than $\alpha_{l}$, the average number of background photons (stray signal photons). Therefore, we have to consider only the effect of dead time due to the gated signal photons. Because the arrival time of stray signal photons at the optical shutter has a uniform distribution, the ratio of lost signals of background photons through the closed shutter to that through the open shutter is given as follows:

$$
\frac{\eta_{\mathrm{sh}} \tau_{d}}{\tau_{\mathrm{sh}}},
$$

where $\eta_{\mathrm{sh}}, \tau_{d}$, and $\tau_{\mathrm{sh}}$ are the average transmittance of the closed shutter, the average dead time, and the average shutter open time, respectively. In our experiment, this ratio is 0.03 when we substitute our experimental parameters: $\eta_{\mathrm{sh}}=0.001, \tau_{d}=1.5 \times 10^{-6}$, and $\tau_{\mathrm{sh}}=33 \times 10^{-9}$. Therefore, we can conclude that we have to consider only the effect of dead time on the background photons through the open shutter.

In the following, we reconstruct the original $\left\{P^{\prime}(n)\right\}$ without loss due to dead time from the experimentally measured $\{P(n)\}$, which can be written using the distribu- 
tions of signal pulses during the shutter open time $\left\{P_{o}(n)\right\}$ and during the shutter closed time $\left\{P_{c}(n)\right\}$ as follows:

$$
P(n)=\sum_{m \leqslant n} P_{o}(n-m) P_{c}(m) .
$$

Here, $\left\{P_{c}(n)\right\}$ can be assumed to have a Poisson distribution with an average photon number of $\left\langle P_{c}(n)\right\rangle$ because the corresponding signals are due to cw-pumped parametric fluorescence:

$$
P_{c}(n)=\exp \left[-\left\langle P_{c}(n)\right\rangle\right]\left\langle P_{c}(n)\right\rangle^{n} / n ! .
$$

For $\left\{P_{o}(n)\right\}$, the probability of counting more than two signal pulses in the shutter open time should be 0 in our experiment:

$$
P_{o}(n \geqslant 2)=0 \text {. }
$$

This is because the dead time $(1.5 \mu \mathrm{s})$ is much larger than the shutter open time (33 ns). Using Eqs. (A2)-(A4), we calculate $\left\langle P_{c}(n)\right\rangle,\left\{P_{o}(0)\right\}$, and $\left\{P_{o}(1)\right\}$ as follows:

$$
\begin{gathered}
\left\langle P_{c}(n)\right\rangle=\frac{P(1)}{P(0)}-\sqrt{\left[\frac{P(1)}{P(0)}\right]^{2}-2 \frac{P(2)}{P(0)}}, \\
P_{o}(0)=\exp \left[\left\langle P_{c}(n)\right\rangle\right] P(0), \\
P_{o}(1)=1-P_{o}(0) .
\end{gathered}
$$

Next, let us consider the relationship between $\left\{P_{o}(n)\right\}$ and the original $\left\{P_{o}^{\prime}(n)\right\}$, which is the signal pulse distribution during the shutter open time when the effect of dead time is compensated. Note that when the signal pulse during the shutter open time eliminates the other signal pulses the number of remaining pulses should still be larger than 1 . Therefore, we have the following relationship:

$$
P_{o}(0)=P_{o}^{\prime}(0) \text {. }
$$

Here, $\left\{P_{o}^{\prime}(n)\right\}$ can be decomposed into two signal pulse distributions. One is the distribution $\left\{P_{\mathrm{G}}(n)\right\}$ of the pulses due to signal photons that are the partners of the firstdetected idler photons during the optical pulse duration, and the other $\left\{P_{\mathrm{BG}}(n)\right\}$ is that of background parametric fluorescence. By definition, these three distributions should be related as follows:

$$
\begin{gathered}
P_{o}^{\prime}(0)=P_{\mathrm{G}}(0) P_{\mathrm{BG}}(0), \\
P_{o}^{\prime}(1)=P_{\mathrm{G}}(0) P_{\mathrm{BG}}(1)+P_{\mathrm{G}}(1) P_{\mathrm{BG}}(0), \\
P_{o}^{\prime}(2)=P_{\mathrm{G}}(0) P_{\mathrm{BG}}(2)+P_{\mathrm{G}}(1) P_{\mathrm{BG}}(1) .
\end{gathered}
$$

In the last equation, we used $P_{\mathrm{G}}(n \geqslant 2)=0$ because the number of detection pulses of the first-detected idler photons should be less than 1 in one optical pulse.

Here, $\left\{P_{\mathrm{BG}}(n)\right\}$ should be a Poisson distribution because the corresponding signals are due to background cwpumped parametric fluorescence. One can estimate the average value of $\left\langle P_{\mathrm{BG}}(n)\right\rangle$ by experimentally measuring the background parametric fluorescence with the shutter open.
Using Eqs. (A8) and (A9), one can give the relationship between $\left\{P_{o}(n)\right\}$ and the original $\left\{P_{o}^{\prime}(n)\right\}$ as follows:

$$
P_{o}^{\prime}(0)=P_{o}(0)
$$

$$
\begin{aligned}
P_{o}^{\prime}(1)= & P_{o}(0)\left\langle P_{\mathrm{BG}}(n)\right\rangle \\
& +\left\{1-P_{o}(0) / \exp \left[-\left\langle P_{\mathrm{BG}}(n)\right\rangle\right]\right\} \exp \left[-\left\langle P_{\mathrm{BG}}(n)\right\rangle\right], \\
P_{o}^{\prime}(2)= & P_{o}(0) \frac{\left\langle P_{\mathrm{BG}}(n)\right\rangle^{2}}{2}+\left\{1-P_{o}(0) / \exp \left[-\left\langle P_{\mathrm{BG}}(n)\right\rangle\right]\right\}, \\
& \times\left\langle P_{\mathrm{BG}}(n)\right\rangle \exp \left[-\left\langle P_{\mathrm{BG}}(n)\right\rangle\right] .
\end{aligned}
$$

Finally, $\left\{P^{\prime}(n)\right\}$ can be calculated only from $\{P(n)\}$ and $\left\langle P_{\mathrm{BG}}(n)\right\rangle$ by using $\left\{P_{c}(n)\right\}$ derived in Eq. (A5) and $\left\{P_{o}^{\prime}(n)\right\}$ in Eq. (A10) as follows:

$$
P^{\prime}(n)=\sum_{m \leqslant n} P_{o}^{\prime}(n-m) P_{c}(m),
$$

where $n \leqslant 2$ in our experiment.

\section{ACKNOWLEDGMENTS}

We thank K. Tsujino for the useful discussion and assistance; K. Ushizaka for assistance with the gate control circuit; and H. F. Hofmann, H. Fujiwara, A. Soujaeff, J. Hotta, and H. Ohashi for the discussion. This research was supported in part by the Japan Science and Technology Corporation-Precursory Research for Embryonic Science and Technology Project, Mitsubishi Electric, and the International Communications Foundation and by a Grant-in-Aid for Scientific Research(B) 12555008 from Japan Society for the Promotion of Science and a Grantin-Aid for the 21st Century Center of Excellence "Topological Science and Technology" Scientific Research from the Ministry of Education, Culture, Sport, Science and Technology of Japan.

R. Okamoto, the corresponding author, can be reached by e-mail at oka@es.hokudai.ac.jp.

\section{REFERENCES}

1. C. H. Bennett and G. Brassard, "Quantum cryptography: public key distribution and coin tossing," in Proceedings of IEEE International Conference on Computers, Systems and Signal Processing (IEEE, 1984), p. 175.

2. E. Knill, R. Laflamme, and G. J. Milburn, "A scheme for efficient quantum computation with linear optics," Nature 409, 46-52 (2001).

3. F. De Martini, G. Di Giuseppe, and M. Marrocco, "Singlemode generation of quantum photon states by excited single molecules in a microcavity trap," Phys. Rev. Lett. 76, 900-903 (1996).

4. F. Treussart, R. Alleaume, V. Le Floc'h, L. T. Xiao, J. M. Courty, and J. F. Roch, "Direct measurement of the photon statistics of a triggered single photon source," Phys. Rev. Lett. 89, 093601 (2002).

5. C. Kurtsiefer, S. Mayer, P. Zarda, and H. Weinfurter, "Stable solid-state source of single photons," Phys. Rev. Lett. 85, 290-293 (2000).

6. J. Kim, O. Benson, H. Kan, and Y. Yamamoto, "A singlephoton turnstile device,” Nature 397, 500-503 (1999). 
7. M. Pelton, C. Santori, J. Vuckovic, B. Zhang, G. S. Solomon, J. Plant, and Y. Yamamoto, "Efficient source of single photons: a single quantum dot in a micropost microcavity," Phys. Rev. Lett. 89, 233602 (2002).

8. C. K. Hong and L. Mandel, "Experimental realization of a localized one-photon state," Phys. Rev. Lett. 56, 58-60 (1986)

9. H. P. Yuen, "Generation, detection, and application of highintensity photon-number-eigenstate fields," Phys. Rev. Lett. 56, 2176-2179 (1986).

10. P. R. Tapster, J. G. Rarity, and J. S. Satchell, "Use of parametric down-conversion to generate sub-Poissonian light," Phys. Rev. A 37, 2963-2967 (1986).

11. J. G. Rarity, P. R. Tapster, and E. Jakeman, "Observation of sub-Poissonian light in parametric downconversion," Opt. Commun. 62, 201-206 (1987).

12. J. Mertz, A. Heidmann, C. Fabre, E. Giacobino, and S. Reynaud, "Observation of high-intensity sub-Poissonian light using an optical parametric oscillator," Phys. Rev. Lett. 64, 2897-2900 (1990).

13. S. Fasel, O. Alibart, S. Tanzilli, P. Baldi, A. Beveratos, Ni. Gisin, and H. Zbinden, "High-quality asynchronous heralded single-photon source at telecom wavelength," New J. Phys. 6, 1631-11 (2004).

14. T. B. Pittman, B. C Jacobs, and J. D. Franson, "Heralding single photons from pulsed parametric down-conversion," Opt. Commun. 246, 545-550 (2004).

15. A. B. U'Ren, C. Silberhorn, K. Banaszek, and I. A. Walmsley, "Efficient conditional preparation of high-fidelity single photon states for fiber-optic quantum networks," Phys. Rev. Lett. 93, 093601 (2004).

16. S. Takeuchi, "Beamlike twin-photon generation by use of type II parametric down conversion," Opt. Lett. 26, $843-845$ (2001).

17. S. Takeuchi, R. Okamoto, and K. Sasaki, "High-yield single-photon source using gated spontaneous parametric downconversion," Appl. Opt. 43, 5708-5711 (2004)

18. C. H. Monken, P. H. S. Ribeiro, and S. Padua, "Optimizing the photon pair collection efficiency: a step toward a loophole-free Bell's inequalities experiment," Phys. Rev. A 57, R2267 (1998).

19. P. G. Kwiat, A. M. Steinberg, R. Y. Chiao, P. H. Eberhard, and M. D. Petroff, "High-efficiency single-photon detectors," Phys. Rev. A 48, R867 (1993).

20. P. R. Tapster and J. G. Rarity, "Photon statistics of pulsed parametric light," J. Mod. Opt. 45, 595-604 (1998).

21. A. L. Migdall, R. U. Datla, A. V. Sergienko, J. S. Orszak, and Y. H. Shih, "Absolute detector quantum-efficiency measurements using correlated photons," Metrologia 32, 479-483 (1995/96). 